\title{
Risk factors for acute psychiatric readmission
}

\author{
Mandy Dixon, Emma Robertson, Mohan George and Femi Oyebode
}

\begin{abstract}
A retrospective case note study explored readmissions to an acute psychiatric in-pationt unit within six months of discharge. The study aimed to calculate a hospltal readmission rate, to investigate the timing of readmissions, and to identify risk factors associated with readmisston. The readmission rate was $27 \%$ with the majority of readmissions occurring within three months after discharge, suggesting the need for investigation of such early readmissions. The three factors found to predict readmission were: discharge against medical advice, number of previous admissions, and living alone or with family rather than in care. Implications for hospital service planning are considered.
\end{abstract}

The closure of psychiatric hospitals in Britain has been accompanied by an increase in community care for the mentally ill. Deinstitutionalisation has led to progressively shorter hospital stays and has raised concern for patient welfare after discharge (Thornicroft \& Bebbington, 1989). With patients discharged from psychiatric facilities now expected to be maintained in the community, readmission has become one of many outcome indicators used to assess the effectiveness of psychiatric services. Being both costly to the services and disruptive to the patient, readmission is usually interpreted as a failure in the mental health system. It is, however, a multifaceted phenomenon caused by a wide range of factors (Rosenblatt \& Mayer, 1974).

Research on readmission has frequently taken the form of discharge follow-up investigations, attempting to isolate demographic or psychiatric risk factors for readmission, but results have been inconclusive. Apart from one consistent risk factor, 'number of previous psychiatric admissions' (Rosenblatt \& Mayer, 1974; Avison \& Speechley, 1987), it is difficult to draw general conclusions from these studies. Most research of this nature has been conducted in the USA, and problems include variable follow-up periods, inconsistent operational definitions, small sample sizes, and diverse study populations, which range from acute to long-term institutions.

The present study investigated readmissions to an acute psychiatric hospital in Birmingham that was opened in the early 1990s and now serves a larger population than originally intended. This has fuelled concern regarding the quality of health care delivery within a "high turnover' environment and has prompted an investigation of the hospital's readmissions, the aim being to identify those patients who are vulnerable to readmission, and to consider appropriate intervention strategies.

The study had four related aims. The first was to calculate a reliable hospital readmission rate. Despite problems of utilising readmission rates as outcome measures (Solomon \& Doll, 1979), readmission rates remain valuable as a crude index of a hospital's discharge outcome. The second aim was to investigate the timing of readmissions, which may inform future intervention strategies. The third was to identify differences in demographic and psychiatric variables between readmitted and non-readmitted patients. Finally, the study aimed to identify risk factors most predictive of readmission to hospital within six months of discharge. The study had the approval of the Local Research Ethics Committee.

\section{The study}

The hospital is an inner-city teaching facility with 162 acute beds, of which 84 are assigned to the Adult Directorate. Patients discharged from the Adult Directorate within a selected fourmonth period $(n=328)$ were identified as the index group. All patients from this index group who were readmitted within six months from their index discharge date $(n=88)$ were classified as readmissions, while those not readmitted within this period were classified as 'non-readmissions' $(n=240)$. Data from the 88 readmissions were then used to describe the timing and rate of readmission. Information necessary for more detailed statistical analysis was available from 78 readmissions, as case notes for the other 10 were reported 'lost'. These 10 did not differ significantly from the sample, and were excluded from subsequent analysis. An equal sample of 78 subjects was randomly drawn from the non-readmissions group to enable a comparative analysis. Random sample selection 
should have ensured that the non-readmission sample was representative of the broader patient population. The total sample consisted of 156 subjects.

Data relating to the following demographic and psychiatric variables were obtained from patients' case notes: age, gender, marital status, ethnicity, occupation, living arrangements, number of previous admissions, diagnosis, length of previous hospital stay, number of referrals made on discharge and whether or not the index discharge was made against medical advice. Information regarding medication compliance and community follow-up was not reliably recorded in the notes and was therefore excluded from the study. The groups were compared on the basis of these variables to identify factors significantly associated with readmission, and, using logistic regression analysis, a model of risk factors predictive of readmission was constructed.

\section{Findings}

Of the total 328 patients discharged, 88 were readmitted within six months, yielding a readmission rate of $27 \%$. Each patient was counted only once in the calculation. If we include in the numerator all admissions of patients who were readmitted more than once within the study period, the readmission rate increases to $29 \%$.

The period between discharge and readmission ranged from 1 to 185 days, with a mean of 77 days (s.d.=56). As illustrated in Fig. 1, the largest proportion of readmissions occurred within the first month after discharge. Moreover, $63.6 \%$ of the readmissions (17\% of all discharges) occurred within three months and could thus be classified as "early readmissions" (Boydel et al, 1991).

The readmission and non-readmission groups were then described and compared. Inferential statistical analysis of the data showed that the following variables were not significantly associated with readmission: gender, age, age at first

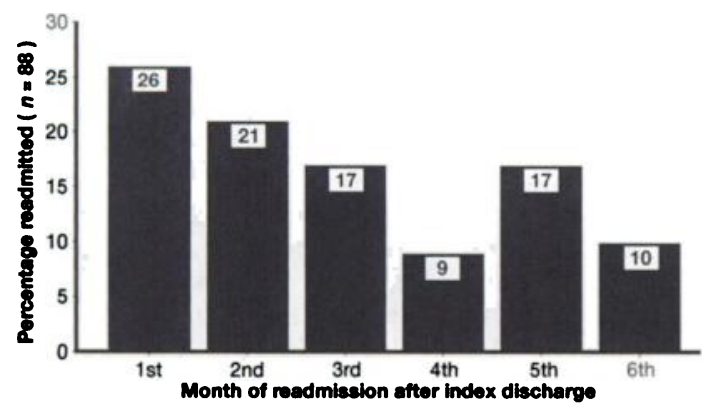

Figure 1. Timing of readmissions. psychiatric admission, number of referrals made on discharge, diagnosis, marital status, ethnic origin and length of previous hospital stay.

The following factors were found to be significantly associated with readmission: living status $\left(\chi^{2}=9.41\right.$, d.f. $\left.=1, P<0.005\right)$ and previous discharge against medical advice $\left(\chi^{2}=9.41\right.$, d.f. $=1, P<0.005$ ). Of the 29 patients who were discharged against medical advice on their index discharge, 22 were subsequently readmitted. two of these were discharged by a Mental Health Review Tribunal and both were readmitted. Patients discharged against advice were also readmitted within a significantly shorter period of time (mean $=50.2$ days, s.d. $=48.9$ ) than those discharged with medical approval (mean $=86.8$ days, s.d. $=57.5 ; t=-2.78$, d.f. $=46.22$, two-tailed $P<0.01)$. There was also a significant difference between the two groups in terms of their mean number of previous psychiatric admissions ( $t=3.02$, one-tailed $P<0.005$ ).

A stepwise logistic regression procedure (SPSS Advanced Statistics for Windows, 1995) was carried out in order to build a model of the factors that are statistically significant predictors of readmission. Likelihood Ratio tests revealed three factors which were associated with a significant increase in the chance of a patient being readmitted $\left(\chi^{2}=22.6\right.$, d.f. $\left.=3, P<0.0001\right)$. These were: taking discharge against medical advice, a higher number of previous psychiatric admissions, and living alone or with one's family. as opposed to living in care. The latter dichotomy was created during the process of analysis. We found that a combination of the variables living alone and living with family yielded a more effective predictive model. Using the above model, $61 \%$ of readmissions and $71 \%$ of nonreadmissions would have been correctly predicted.

\section{Comment}

Controversy surrounds the use of readmission rates as a measure of service effectiveness. This paper reports the figure only as a crude index of the number of discharges who return to hospital during a given period. Our figure of $27 \%$ is comparable to readmission rates reported elsewhere; Solomon et al (1984) reported an identical figure for their study of readmissions within a six month period, while the Audit Commission reported a range of 9-25\% with a mean of $16 \%$ (personal communication, 1995).

Most of the readmissions occurred within the first three months after discharge (Fig. 1). These 'early readmissions', also labelled "failed discharges" (Jones, 1991) may indicate a need for closer investigation of discharge policy and 
practice, or indeed the notion of a failed intervention by the hospital.

The most consistent predictor of readmission found in the literature has been the patients number of previous admissions. Similarly, this study found that patients who were readmitted to hospital had a greater number of prior hospitalisations than those who did not return. Clearly, the association of this variable with readmission masks a complex array of circumstances, and future studies may benefit by controlling for number of previous admissions. This may pose difficulties as this information is not routinely collected. Attempts to identify the level at which the number of previous admissions is most strongly associated with readmissions have proved difficult. Careful inspection of our data, however, suggests that patients with 10 or more prior hospitalisations are at greatest risk of readmission.

There was no significant difference between the two groups in terms of age at first psychiatric admission. This may suggest that it is the contact with in-patient services, rather than the duration of the illness, that predisposes someone to be readmitted. As Gillis et al (1985) have suggested: "once the channel to hospital has been opened by previous contact it becomes a ready resource when troubles arise at home or in the community". Therefore, as patients have an increasing number of admissions, it perhaps becomes easier for them to be readmitted during times of crisis.

This could denote an interaction with the other predictive factor: living with family or alone. In times of crisis, family living with patients who have a history of frequent contact with the hospital may use these established links to organise the patient's readmission. The appropriateness of these readmissions needs to be explored. While the family may serve a useful purpose in identifying and supporting the individual in times of crisis, perhaps other alternatives to hospital admission could be presented to the family at such times. Conversely, hostilities within the family environment could be instrumental in the individual's continuing hospital admission, and intervention should address this problem, particularly if admissions represent crisis or intolerance within the family, rather than a patient's illness (Falloon et al. 1983).

The association between readmission and living alone is perhaps more apparent, suggesting poor support and social isolation. It has been suggested that a reciprocal relationship exists between an individual's hospitalisation patterns and their social networks (Holmes-Eber \& Riger, 1990). The stigma and disruption associated with multiple admissions affects the composition of an individual's social networks, to the extent that they may live alone with little social support outside of the mental health services. This creates a situation in which the individual is increasingly dependent on the services and is therefore more likely to be admitted to hospital during times of crisis. The importance of social networks to patients' hospital utilisation therefore needs to be recognised in any intervention that is designed to reduce multiple admissions.

There is a dearth of studies in British psychiatry exploring discharge from hospital against medical advice (AMA), which emerged as the strongest predictor of readmission in this study. Of the discharges included in the analysis, 20\% were AMA. Whereas little comparative information exists in Britain, Canadian figures suggest that between 6 and 35\% of psychiatric patients discharge themselves from hospital AMA (Dalrymple \& Fata, 1993). A link between discharge AMA and multiple psychiatric admissions has been suggested by Evans et al (1992), who found that 'revolving door' patients were associated with discharge AMA. They suggest that once a patient becomes known as a 'revolving door' patient, this may influence staff attitudes to them. This would have repercussions, both on the ward where it may increase the likelihood of patients discharging themselves AMA, and in the community, where it may increase the chances of a patient being readmitted. While further research is needed to explore this complex relationship, our results clearly suggest that patients who discharge themselves not only increase their risk of readmission, but tend to be readmitted sooner than patients who are discharged with medical approval.

In terms of hospital policy, without debating the ethics of involuntary detention, we can recommend that hospitals should focus interventions for this vulnerable group on their postdischarge follow-up. In this study, those who self-discharged and were then readmitted were given fewer referrals on discharge than those who were not subsequently readmitted. Although this difference was not statistically significant, and must be interpreted cautiously, hospitals should perhaps intensify their support programmes for these individuals, particularly in the period immediately following discharge.

The findings of this study have several implications for hospital policy. Patients who have a higher number of prior admissions, who live alone or with family, and who are discharged prematurely, are at particular risk for readmission. As most readmissions seem to occur within the first few months after discharge, interventions designed to prevent readmissions should focus on the period immediately following discharge, perhaps instituting intensive follow-up programmes for the high 
risk group identified above. The issue of whether or not the readmissions could have been prevented is, of course, central to service providers who wish to reduce avoidable readmissions. A prospective study is currently being designed to focus on early readmissions and to consider the issue of whether or not they could have been prevented.

\section{Acknowledgements}

The authors thank Mr Roger Holder from the University of Birmingham's Department of Mathematics and Statistics for his advice and assistance. The study was funded by the West Midlands Regional Health Authority as one of their Nursing and Therapy Audit projects, and thanks go to Mrs Maria Sagar for securing this funding.

\section{References}

AVISON, W. R. \& SPEECHLEY, K. N. (1987) The discharged psychiatric patient: a review of social, socialpsychological, and psychiatric correlates of outcome American Journal of Psychiatry, 144, 10-18.

BoYdel, K. M., MALCOLMSON. S. A. \& SiKerBol, K. (1991) Early rehospitalization. Canadian Journal of Psychiatry. 36. 743-745.

DALRYMPLE, A. J. \& FATA, M. (1993) Cross validating factors associated with discharges against medical advice. Canadian Journal of Psychiatry. 38. 285-259.

Evans, M., RICE, D. \& RouTH, C. (1992) Patients repeatedly admitted to psychiatric wards. Psychiatric Bulletin, 16. 157-158.
FALLOON, R. H., MARShaLl, G. N., BOYD, J. L., et al (1983) Relapse in schizophrenia: a review of the concept and its definitions. Psychological Medicine, 13, 469-477.

GILUS, L. S., SANDLER, R., JAKOET, A., et al (1985) Outcome after discharge from a psychiatric hospital. South African Medical Joumal, 68, 470-472.

HOLMES-EBER, P. \& RIGER, S. (1990) Hospitalization and the composition of mental patients' social networks. Schizophrenia Bulletin, 16, 157-164.

JONES, E. (1991) Audit in psychiatry: failed discharges. Psychiatric Bulletin, 15, 26-27.

ROSENBLATT, A. \& MAYER, J. E. (1974) The recidivism of mental patients: a review of past studies. American Journal of Orthopsychiatry. 44, 697-705.

SOLOMON, P. \& DoL. W. (1979) The varieties of readmission: The case against the use of recidivism rates as a measure of program effectiveness. American Journal of Orthopsychiatry, 49, 230-239.

-. GORDON, B. \& DAVIS, J. (1984) Differentiating psychiatric readmissions from nonreadmissions. American Journal of Orthopsychiatry. 54, 426-435.

THORNICROFT, G. \& BEBBington. P. (1989) Deinstitutionalisation - from hospital closure to service development. British Journal of Psychiatry. 165. 739753.

Mandy Dixon, Research Manager, Emma Robertson, Research Associate; Mohan George, Consultant Psychiatrist, and Femi Oyebode. Consultant Psychiatrist, Queen Elizabeth Psychiatric Hospital, Mindelsohn Way, Edgbaston, Birmingham B15 2QZ

*Correspondence 